\title{
Computed tomography-based body composition profile as a screening tool for geriatric frailty detection
}

\author{
Olga Laur ${ }^{1}$ - Michael J. Weaver ${ }^{2} \cdot$ Christopher Bridge $^{3} \cdot$ Erika Chow $^{7} \cdot$ Michael Rosenthal $^{4} \cdot$ Camden Bay $^{7}$. \\ Houman Javedan ${ }^{5} \cdot$ Mitchel B. Harris $^{6} \cdot$ Bharti Khurana $^{7}$
}

Received: 6 June 2021 / Revised: 31 October 2021 / Accepted: 31 October 2021 / Published online: 4 December 2021

(c) ISS 2021

\begin{abstract}
Objective To assess prevalence of CT imaging-derived sarcopenia, osteoporosis, and visceral obesity in clinically frail and prefrail patients and determine their association with the diagnosis of frailty.

Materials and methods This cross-sectional study was constructed using our institution's pelvic trauma registry and ambulatory database registry. The study included all elderly pelvic trauma patients and ambulatory outpatients between May 2016 and March 2020 who had a comprehensive geriatric assessment and CT abdomen/pelvis within 1 year from the date of the assessment. Patients were dichotomized in prefrail or frail groups. The study excluded patients with history of metastatic disease or malignancy requiring chemotherapy.

Results The study cohort consisted of 151 elderly female and 65 male patients. Each gender population was subdivided into frail (114 female [75\%], 51 male [78\%]) and prefrail (37 female [25\%], 14 male [22\%]) patients. CT-imaging-derived diagnosis of osteoporosis (odds ratio, 2.5; 95\% CI: 1.2-5.5) and sarcopenia (odds ratio, 2.6; 95\% CI: 1.2-5.6) were associated with frailty in females, but did not reach statistical significance in males. BMI and subcutaneous adipose tissue at L3 level were statistically lower in the frail male group compared to the prefrail group. BMI showed strong correlation with the subcutaneous area at the L3 level in both genders (Spearman's coefficient of $0.8, p<0.001$ ). Hypoalbuminemia and visceral obesity were not associated with frailty in either gender.

Conclusion This proof-of-concept study demonstrates the feasibility of using CT-derived body-composition parameters as a screening tool for frailty, which can offer an opportunity for early medical intervention.
\end{abstract}

Keywords Frailty $\cdot$ Body composition $\cdot$ Machine learning

Olga Laur

olgaolaur@gmail.com

1 Department of Radiology, New York Presbyterian Hospital, Weill Cornell Medicine, 525 East 68(th) Street, New York, NY 10065, USA

2 Department of Orthopedic Surgery, Brigham and Women's Hospital, Boston, MA, USA

3 MGH \& BWH Center for Clinical Data Science, Boston, MA, USA

4 Department of Radiology, Dana Farber Cancer Institute, Boston, MA, USA

5 Division of Aging, Department of Medicine, Brigham and Women's Hospital, Boston, MA, USA

6 Department of Orthopedics, Massachusetts General Hospital, Boston, MA, USA

7 Department of Radiology, Division of Emergency Radiology, Brigham and Women's Hospital, Boston, MA, USA

\section{Introduction}

Frailty represents a dynamic multidimensional syndrome or state characterized by increased vulnerability to outside stressors secondary to aging-related degeneration of physiologic reserve and psychologic and social functioning [1]. Frailty approximates patient's biologic age - an active rate at which body is aging - which may be more relevant to the clinical practice compared to the chronological age. Frail patients display increased vulnerability to adverse health outcomes, which have been shown to be independent of age, comorbidity, and disability [2-5]. Frailty comes at a high cost to society, as frail patients are at increased risk of fractures, more likely to be discharged to a skilled nursing facility, more likely to be readmitted within 30 days, and have higher rates of postoperative mortality and complications compared to their robust elderly peers [6-11]. 
According to the United States Census Bureau, 1 in every 5 Americans is projected to be older than 65 by the year 2030, with recent meta-analysis demonstrating the prevalence of frailty up to $18 \%$ in this age group and a significant increase in prevalence among those older than 85 years, especially in non-community dwelling older adults $[12,13]$. Concurrently, the American College of Surgeons' national trauma databank has reported that adults of age 65 and older constitute $30.75 \%$ of all trauma-related incidents evaluated in the emergency department by the surgical team [14]. Falls, which commonly occur with frailty, represented the most common mechanism of injury, with the incidence increasing with age. This evidence suggests that frailty is common and its prompt diagnosis is necessary for prevention of adverse events and appropriate clinical management.

Assessment of frailty has traditionally been accomplished via a comprehensive geriatric assessment $[1,15]$. During this assessment, patients are queried on how they move, function, think, medication use, and social support, among other factors. At the end of the assessment, frailty status can be determined by a more qualitative clinical frailty score (CFS) or a more quantitative and precise frailty index (FI) score, which recently entered clinical practice $[15,16]$.

Currently, frailty is underdiagnosed [5]. Lack of time is a major barrier to frailty screening, particularly at the frontline of healthcare such as busy emergency department or primary care physicians office with a heavy patient load [17]. Access to a dedicated geriatric specialist represents another limiting factor. Additionally, elderly patients are often considered to be "difficult" interviewers secondary to a variety of factors such as deafness or dementia. Often, additional corroborative information from the relatives and caretakers is necessary to make an accurate assessment.

Abdominal CT imaging may provide valuable insights into patients' body composition by assessing the characteristics of the muscle, adipose tissue, and bone. These factors may represent surrogate markers of frailty, with prior studies showing osteopenia and sarcopenia being associated with a higher risk of mortality in elderly patients, although their frailty status was not assessed by a comprehensive geriatric evaluation $[18,19]$. If feasible, automated flagging of patients at risk of frailty provides multiple inherent advantages because it may be achieved in an instantaneous manner, does not rely on patient interviewing, and uses information obtained from an already existing study at no extra cost to society.

To our knowledge, this is the first study assessing CTderived imaging parameters of the adipose tissue, muscle, and bone in patients who had a clinical diagnosis of frailty or prefrailty assigned after a comprehensive geriatric assessment with a dedicated full physical exam and comprehensive history taking, which represents gold standard in frailty assessment as opposed to simplified questionnaires or retrospective chart review for frailty approximation. Our primary objective was to assess prevalence of CT imaging-derived sarcopenia, osteoporosis, and visceral obesity in clinically frail and prefrail patients and determine their association with the clinical diagnosis of frailty. We hypothesized that frail patients had a readily recognizable imaging phenotype characterized by osteopenia, sarcopenia, and visceral obesity.

\section{Materials and methods}

This retrospective study was approved by the institutional review board and compliant with the Health Insurance Portability and Accountability Act. This study was not supported by the industry.

\section{Study design and human subjects}

In our analysis, men and women underwent an independent cohort analysis given known differences in their body composition [20]. Subject baseline characteristics were described separately for prefrail and frail groups. CT imaging was processed by an automated machine-learning model developed at our institution, which determined the total cross-sectional area of the muscle, visceral, and subcutaneous adipose tissue at third lumbar vertebral level (L3) for each patient (Fig. 1) [21, 22]. Briefly, the machine learning model by Bridge et al. auto-segmented body composition parameters at L3 vertebral level by utilizing a 2-step process using a DenseNet convolutional neural network to select the appropriate CT slice, followed by U-Net convolutional neural network to perform segmentation. This model automatically analyzed aforementioned body composition parameters in a fraction of a second, which would be impossible with manual segmentation. Of note, vertebral body attenuation of L3 was measured manually as it was not automated yet at the time of data collection.

\section{Data source}

Using our institution's trauma registry, we identified 211 consecutive patients of 65 years or older who were admitted with pelvic fractures between May 2016 and October 2019. In addition, we queried the institutional database registry from August 2016 to March 2020 for all ambulatory patients who had the "clinical frailty scale" phrase present in their ambulatory report and had CT imaging in their record. Two hundred sixteen patients constituted the final cohort (102 trauma patients and 114 ambulatory patients) (Fig. 2). Inclusion criteria for both cohorts included comprehensive geriatric assessment and $\mathrm{CT}$ abdomen/pelvis imaging performed within 1 year from the time of the geriatric 
Fig. 1 a Axial CT imaging slice at L3 vertebral level taken from a CT abdomen-pelvis with $\mathbf{b}$ corresponding color-coded total cross-sectional area of the muscle (red), visceral (yellow), and subcutaneous (green) adipose tissue which was determined by the automated machine learning segmentation model developed at our institution

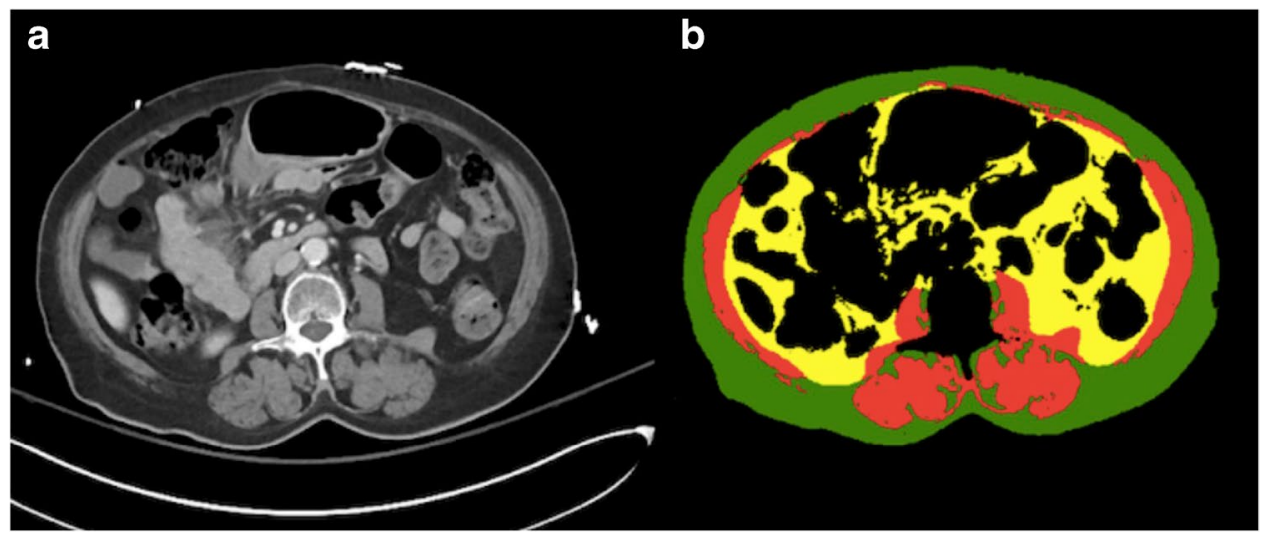

Ambulatory cohort:

Trauma cohort:

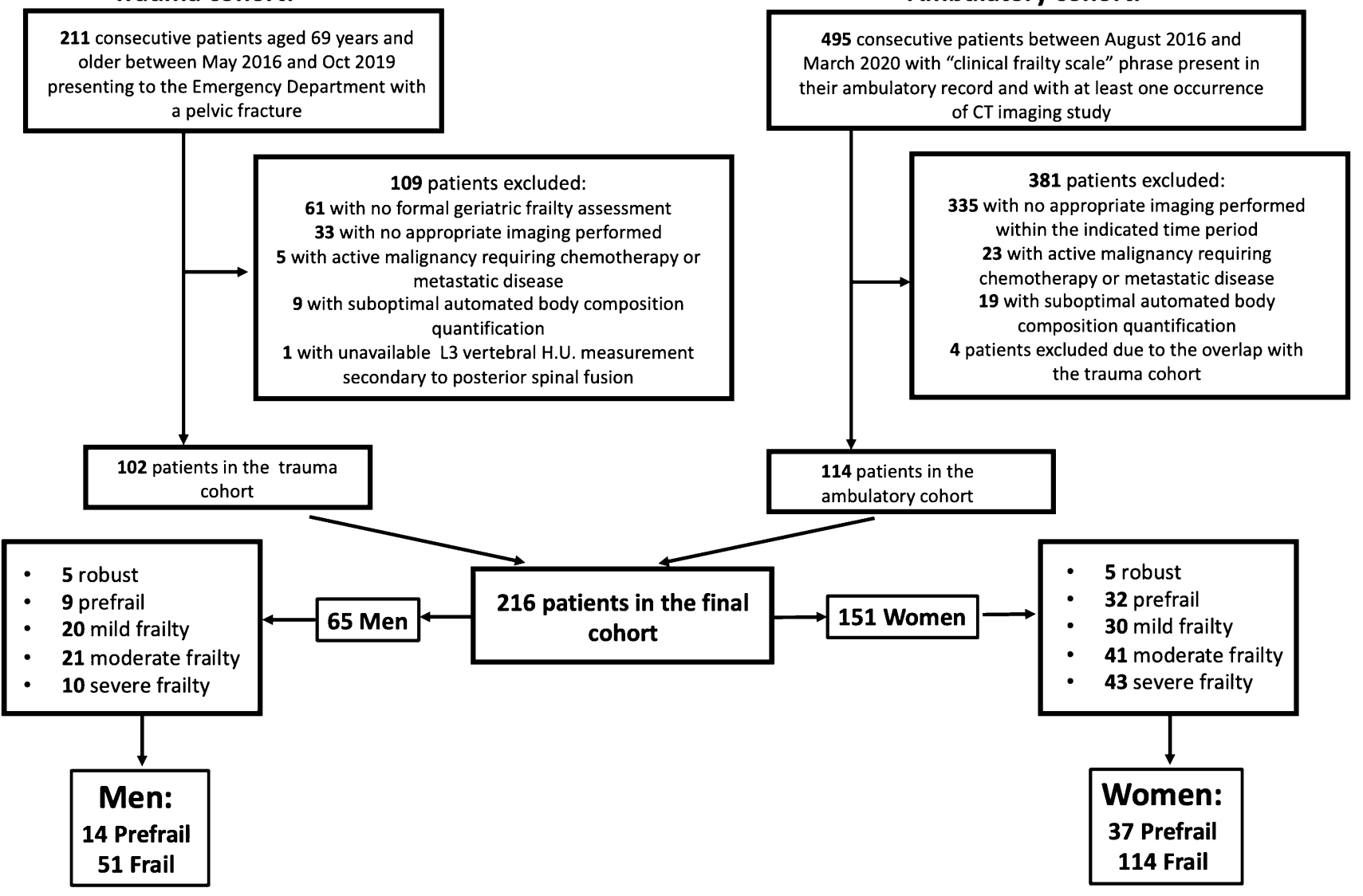

Fig. 2 Study participant flow diagram

evaluation regardless of the indication. Exclusion criteria included history of metastatic disease or active malignancy requiring chemotherapy, suboptimal automated body composition quantification as determined by manual review (for example, an arm overlying the abdominal wall with a prominent streak artifact). Patients with an overlap between trauma and ambulatory cohorts were counted only once.

The Canadian Study on Health and Aging (CSHA) Clinical Frailty Scale (CFS) was assigned to all patients by board certified geriatric medicine physicians in our institution. Briefly, determination of patient's frailty state was based on the reviewer's clinical judgment upon completion of a comprehensive geriatric assessment, which among other factors assessed patient's movement status, function, thought process, medication use, social history, and personal assessment of health, with frequent corroborative information from the relatives and caretakers. The Clinical Frailty Scale (CFS) was used to assess patient's clinical frailty status until 2018 
at our institution. CFS represented a qualitative approach of summarizing the key points of comprehensive geriatric assessment and was primarily based on the deficits in the functional category of activities of daily living (ADLs) and instrumental activities of daily living (IADLs). Starting year 2018, CSF has been replaced by the frailty index (FI) score at our institution, which instead of qualitative assessment provided quantitative measurement of all of the patient's deficits and believed to be the best tool in relaying patient's frailty status [15]. In these instances, the numeric value of FI score was converted to CFS using established conversion criteria [23]. Using CFS, patients were stratified into the well, prefrail, mild, moderate, and severely frail groups (Fig. 2). When dichotomizing groups, well and prefrail patients were grouped into prefrail group, and the rest of the patients were grouped into the frail group.

\section{Data extraction}

The electronic medical record was reviewed for demographic information, including age, sex, and race, as well as biometric and laboratory parameters such as height, weight, BMI, and albumin level. Race of 17 patients (7.8\%) as well as albumin level for 1 patient $(0.4 \%)$ was unavailable for review. Albumin level of $<3.5 \mathrm{mg} / \mathrm{dL}$ was considered to represent hypoalbuminemia [24]. Other indicators for the trauma cohort $(n=102,47.2 \%)$ including injury severity score (ISS), total hospital days, total ICU days, and discharge site were extracted from the trauma registry. The degree of medical complexity was evaluated using the Charlson comorbidity index (CCI). Expired patients were identified via medical record review and 1-year mortality from the time of injury was recorded.

\section{Exposure variables}

\section{Sarcopenia}

The automated cross-sectional muscle area measurement at the L3 vertebral level contained psoas, paraspinal, and abdominal wall muscles. Skeletal muscle index (SMI) was defined as a total cross-sectional muscle area at the L3 vertebral level divided by patient's height ${ }^{2}\left(\mathrm{~cm}^{2} / \mathrm{m}^{2}\right)$. Based on prior literature, sarcopenia cutoffs in men and women were determined to be $53.0 \mathrm{~cm}^{2} / \mathrm{m}^{2}$ for men with BMI $\gtrsim 25.0 \mathrm{~kg} /$ $\mathrm{m}^{2}, 43 \mathrm{~cm}^{2} / \mathrm{m}^{2}$ for men with BMI $<25.0 \mathrm{~kg} / \mathrm{m}^{2}$, and 41.0 $\mathrm{cm}^{2} / \mathrm{m}^{2}$ for women regardless of BMI [25].

\section{Osteoporosis}

A $1.5-2 \mathrm{~cm}^{2}$ region of interest was drawn manually over trabecular bone at L3 vertebral level (in 6 cases (2.8\%) of compression deformity or hemangioma, the measurement occurred at the adjacent lumbar level). This was done by two participating radiology residents and one radiologist who were blinded to the results of frailty scoring. A threshold of bone attenuation $<90 \mathrm{HU}$ was chosen to define osteoporosis [26].

\section{Visceral obesity}

All patients were classified into four BMI groups based on the Center for Disease Control guidelines: underweight $\left(\mathrm{BMI}<18.5 \mathrm{~kg} / \mathrm{m}^{2}\right)$, normal $(\mathrm{BMI}>=18.5$ and $<25.0 \mathrm{~kg} /$ $\left.\mathrm{m}^{2}\right)$, overweight $\left(\mathrm{BMI}=25.0-29.9 \mathrm{~kg} / \mathrm{m}^{2}\right)$, and obese $\left(B M I \geq 30 \mathrm{~kg} / \mathrm{m}^{2}\right)$. Visceral adipose tissue (VAT) was defined as an automated measurement of the adipose tissue area at L3 vertebral level and included adipose tissue beneath the abdominal wall. Subcutaneous adipose tissue (SAT) was defined as an automated measurement of the abdominal wall adipose tissue area at the $\mathrm{L} 3$ vertebral level. Visceral obesity ratio (VOR) was measured as a ratio of VAT over SAT. Additionally, visceral obesity was assessed by determining cross-sectional area at L3 level with visceral obesity cutoff $\geq 130 \mathrm{~cm}^{2}$ in men, and $\geq 110 \mathrm{~cm}^{2}$ in women [27-29].

\section{Statistical analysis}

Ordinal or nominal variables were presented as absolute numbers with percentages of the total. Non-parametric data were presented as median with inter quartile range. L3 vertebral body HU, SMI, VAT, and SAT were presented continuously and dichotomously as osteoporosis, sarcopenia, and visceral obesity based on previously published body composition imaging marker cutoffs. Pearson $\chi^{2}$ test or Fisher exact test were used for categorical comparisons, as appropriate. Wilcoxon rank sum test was used for continuous variables. Two-tailed Spearman correlation was used for correlation analysis. To evaluate association with CT-derived body composition markers with frailty, univariate logistic regression analyses were performed, with osteoporosis, sarcopenia, hypoalbuminemia, and visceral obesity as dependent variables and frailty as independent variable. Binary logistic regression was performed for age and cohort status adjustment (trauma vs. ambulatory). Odds ratios (ORs) and 95\% CI were provided. SPSS version 23.0 (SPSS Inc., Chicago, IL, USA) was used for all statistical analysis.

\section{Outcome measures}

Primary Outcome: To determine CT-imaging derived prevalence of sarcopenia, osteoporosis and visceral obesity in frail geriatric patient group compared to the prefrail group and to assess their association with clinical diagnosis of frailty. 
Secondary Outcome: To determine prevalence of hypoalbuminemia in frail geriatric patient group compared to the prefrail group and to assess its association with clinical diagnosis of frailty.

\section{Results}

\section{Baseline characteristics of the study cohort}

The study included 151 female and 65 male patients. Both genders were classified as frail (114 female patients [75\%], 51 male patients [78\%]) or prefrail (37 female patients [25\%], 14 male patients [22\%]) (Fig. 2). There was a statistically higher rate of mortality at 1 year in frail patients compared to prefrail patients (1 prefrail patient [2\%] vs. 23 frail patients [14\%], $p=0.019)$.

Both frail female (years median, IQR 78.0 (71.5-83.5) in prefrail vs 84.0 (76.0-89.0) in frail group, $p=0.004)$ and frail male patients (years median, IQR $76.0(72.8-82.0)$ in prefrail vs $82.0(77.0-88.0)$ in frail group, $p=0.03)$ were slightly older compared to their respective prefrail groups (Table 1). The trauma subset of the female frail patient group also had higher CCI score (CCI score, IQR 4.0 (3.0-5.5) in prefrail vs. $6.0(5.0-7.0)$ in frail group, $p=0.001)$.
Otherwise, no statistically significant difference was identified in terms of demographic features or trauma-related characteristics in the prefrail and frail groups among both genders.

\section{Female CT-derived body composition and anthropomorphic data-frail vs. prefrail}

Frail female patients had a statistically significant lower bone attenuation compared to the prefrail group (HU median, IQR $99.0(76.0-127.0)$ in prefrail vs $75.0(48.5-121.3)$ in frail patients $p=0.03$ ) (Table 1). BMI, skeletal muscle index (SMI), visceral adipose tissue (VAT), subcutaneous adipose tissue (SAT), or albumin levels did not show statistically significant difference between groups. When body composition imaging parameters were dichotomized into groups of CTderived imaging diagnosis of osteoporosis, sarcopenia, and visceral obesity, patients with osteoporosis (odds ratio, 2.5; 95\% CI, 1.2-5.5) and patients with sarcopenia (odds ratio, 2.6 ; 95\% CI, 1.2-5.6) had a statistically significant association with frailty (Table 2). The strength of the relationship between osteoporosis and frailty persisted in females after multivariate adjustment (odds ratio, 3.1; 95\% CI, 1.2-7.8). The strength of the relationship between sarcopenia and frailty decreased slightly following multivariate adjustment
Table 1 Demographic, laboratory, and CT-derived body composition characteristics of prefrail and frail patients stratified by gender. Baseline characteristics labeled with $*$ represent trauma cohort data only
( $n=102,47.2 \%$; prefrail women $n=21$, frail women $n=51$; prefrail men $=9$, frail men $=21$ )

\begin{tabular}{|c|c|c|c|c|c|c|}
\hline \multirow{2}{*}{$\begin{array}{l}\text { Characteristic } \\
\text { Baseline characteristics ( } n \text {, median } \\
(I Q R))\end{array}$} & \multicolumn{2}{|l|}{ Female } & \multirow[t]{2}{*}{$P$ value } & \multicolumn{2}{|l|}{ Male } & \multirow[t]{2}{*}{$p$ value } \\
\hline & Prefrail $(N=37)$ & Frail $(N=114)$ & & Prefrail $(N=14)$ & Frail $(N=51)$ & \\
\hline Age, y & $78.0(71.5-83.5)$ & $84.0(76.0-89.0)$ & 0.004 & $76.0(72.8-82.0)$ & $82.0(77.0-88.0)$ & 0.03 \\
\hline Race (white) $(n, \%)$ & $27(75.0)$ & $78(76.0)$ & 1.0 & $13(92.8)$ & $36(78.3)$ & 0.43 \\
\hline $\begin{array}{l}\text { Less than } 60 \text { days between geriatric } \\
\text { evaluation and CT imaging }(n, \%)\end{array}$ & $26(70.3)$ & $76(66.7)$ & 0.84 & $11(78.6)$ & $36(70.6)$ & 0.80 \\
\hline Injury severity score* & $13.0(8.5-18.0)$ & $13.0(9.0-17.0)$ & 0.70 & $8.0(4.5-19.5)$ & $10.0(4.0-17.0)$ & 0.79 \\
\hline Total hospital days* & $5.0(3.0-6.5)$ & $5.0(4.0-7.0)$ & 0.48 & $6.0(5.5-8.0)$ & $7.0(3.5-10.0)$ & 0.82 \\
\hline Total ICU days* & $2.0(0.0-3.5)$ & $3.0(0.0-4.0)$ & 0.31 & $1.0(0-3.0)$ & $3.0(0-6.0)$ & 0.35 \\
\hline Charlson comorbidity index $(\mathrm{CCI})^{*}$ & $4.0(3.0-5.5)$ & $6.0(5.0-7.0)$ & 0.001 & $5.0(3.5-6.5)$ & $6.0(4.5-7.0)$ & 0.26 \\
\hline Discharge to home $(n, \%)^{*}$ & $4(19.0)$ & $3(5.9)$ & 0.18 & $1(11.1)$ & $0(0.0)$ & 0.30 \\
\hline Albumin (mg/dL) & $4.0(3.2-4.3)$ & $3.7(3.2-4.2)$ & 0.15 & $4.1(3.4-4.2)$ & $3.7(3.0-4.2)$ & 0.34 \\
\hline BMI & $25.8(22.7-28.4)$ & $23.9(20.7-27.8)$ & 0.18 & $27.1(25.5-27.9)$ & $24.0(21.5-26.4)$ & 0.01 \\
\hline $\begin{array}{l}\text { Proportion of obese or underweight } \\
\text { patients based on BMI }(n, \%)\end{array}$ & $7(18.9)$ & $36(31.6)$ & 0.2 & $2(14.2 \%)$ & $10(19.6 \%)$ & 0.83 \\
\hline \multicolumn{7}{|c|}{ CT-derived body composition characteristics } \\
\hline L3 vertebra attenuation (HU) & $99.0(76.0-127.0)$ & $75.0(48.5-121.3)$ & 0.03 & $122.5(92.5-140.3)$ & $97.0(67.0-137.0)$ & 0.15 \\
\hline Skeletal muscle index (SMI), $\mathrm{cm} / \mathrm{m}^{2}$ & $41.6(35.2-45.5)$ & $37.0(32.3-42.8)$ & 0.06 & $47.1(42.9-51.4)$ & $42.3(38.1-49.5)$ & 0.07 \\
\hline Visceral adipose tissue (VAT), $\mathrm{cm}^{2}$ & $118.6(56.5-152.1)$ & $91.4(52.6-172.9)$ & 0.58 & $192.7(133.1-302.8)$ & $155.9(74.3-280.4)$ & 0.35 \\
\hline $\begin{array}{l}\text { Subcutaneous adipose tissue (SAT), } \\
\mathrm{cm}^{2}\end{array}$ & $205.0(160.9-264.8)$ & $203.7(129.3-302.3)$ & 0.63 & $188.8(133.0-221.5)$ & $137.1(102.7-175.9)$ & 0.01 \\
\hline Visceral obesity ratio (VOR) & $0.49(0.27-0.73)$ & $0.51(0.33-0.66)$ & 0.85 & $0.97(0.70-1.60)$ & $1.22(0.74-1.77)$ & 0.54 \\
\hline
\end{tabular}


Table 2 Gender stratified odds ratio of the clinical diagnosis of frailty in relation to CT-derived body composition imaging diagnosis of osteoporosis, sarcopenia, and visceral obesity as well as laboratory diagnosis of hypoalbuminemia in univariate analysis and multivariate analysis adjusted by age and type of cohort (ambulatory vs. trauma)

\begin{tabular}{|c|c|c|c|c|c|c|}
\hline $\begin{array}{l}\text { CT-derived and laboratory } \\
\text { body composition variable }\end{array}$ & Prefrail $(N, \%)$ & Frail $(N, \%)$ & Univariate OR (95\% CI) & $p$ value & Multivariate OR (95\% CI) & $p$ value \\
\hline Female & Prefrail $(N=37)$ & Frail $(N=114)$ & & & & \\
\hline Osteoporosis & $13(35.1)$ & $66(57.9)$ & $2.5(1.2-5.5)$ & 0.02 & $3.1(1.2-7.8)$ & 0.02 \\
\hline Sarcopenia & $16(43.2)$ & $76(66.7)$ & $2.6(1.2-5.6)$ & 0.01 & $2.4(1.0-5.5)$ & 0.04 \\
\hline Visceral obesity & $20(54.1)$ & $44(38.6)$ & $0.5(0.3-1.1)$ & 0.10 & $0.6(0.3-1.3)$ & 0.18 \\
\hline Hypoalbuminemia & $11(30.6)$ & $49(43.0)$ & $1.7(0.8-3.8)$ & 0.19 & $2.2(0.9-5.5)$ & 1.00 \\
\hline Male & Prefrail $(N=14)$ & Frail $(N=51)$ & & & & \\
\hline Osteoporosis & $3(21.4)$ & $22(43.1)$ & $2.8(0.7-11.2)$ & 0.14 & $4.1(0.9-19.3)$ & 0.08 \\
\hline Sarcopenia & $9(64.3)$ & $32(62.7)$ & $0.9(0.3-3.2)$ & 0.92 & $0.9(0.2-3.3)$ & 0.85 \\
\hline Visceral obesity & $11(78.6)$ & $29(56.9)$ & $0.4(0.1-1.5)$ & 0.14 & $0.3(0.1-1.5)$ & 0.15 \\
\hline Hypoalbuminemia & $4(28.6)$ & $21(42.0)$ & $1.8(0.50-6.6)$ & 0.36 & $3.8(0.7-19.4)$ & 0.11 \\
\hline
\end{tabular}

but remained statistically significant (odds ratio, 2.4; $95 \%$ CI, 1.0-5.5).

CT-derived imaging diagnosis of visceral obesity showed no statistically significant association with frailty. Visceral obesity ratio (VOR) demonstrated predominance of subcutaneous over visceral adipose tissue in females in both prefrail and frail groups (VOR median, IQR 0.49 (0.27-0.73) in prefrail vs. 0.51 (IQR 0.33-0.66) in frail patients, $p=0.9$ ). Correlation analysis between CT-derived body composition parameters, laboratory albumin levels, and BMI demonstrated strong correlation between BMI and SAT $(r=0.82$, $p<0.001)$, and moderate correlation between BMI and VAT $(r=0.69, p<0.001)$ and between VAT and SAT $(r=0.67$, $p<0.001$ ) (Table 3).

\section{Male CT-derived body composition and anthropomorphic data-frail vs. prefrail}

Frail male patients had a statistically significant lower BMI compared to the prefrail group (BMI median, IQR 24.0 (21.5-26.4) in frail vs. 27.1 (25.5-27.9) in prefrail group, $p=0.009)$, as well as lower subcutaneous adipose tissue (SAT) (SAT median, IQR 137.1 (102.7-175.9) $\mathrm{cm}^{2}$ in frail group vs. $188.8(144.0-221.5) \mathrm{cm}^{2}$ in prefrail group, $p=0.01$ ) (Table 1). Bone attenuation, SMI, and VAT did not show statistically significant difference between these groups. Dichotomization of CT-derived body composition parameters into groups of imaging-derived diagnosis of osteoporosis, sarcopenia, and visceral obesity showed no statistical association with frailty (Table 2).

Visceral obesity ratio (VOR) in males demonstrated similar to higher visceral to subcutaneous adipose tissue ratio in in both groups (VOR median, IQR 1.22 (0.74-1.77) in frail vs. $0.97(0.70-1.60)$ in prefrail patients, $p=0.5)$, which was in contrast to females. Correlation analysis between CTderived body composition parameters, laboratory albumin levels, and BMI demonstrated strong correlation between BMI and SAT ( $r=0.78, p<0.001)$, and moderate correlation between BMI and VAT $(r=0.67, p<0.001)$ as well as VAT and SAT $(r=0.68, p<0.001)$ (Table 3$)$.

\section{Female and male laboratory albumin levels-frail vs. prefrail}

Albumin levels did not show statistically significant difference between female or male prefrail and frail groups (Table 1). Hypoalbuminemia showed no statistically significant association with frailty (Table 2).

\section{Discussion}

It has been well known that sarcopenia and osteoporosis, as determined by traditional methods of dual-energy X-ray absorptiometry, are prevalent in frail patients [30-33]. To our knowledge, this is the first study to date to assess CTderived body composition profile in patients with a clinical diagnosis of frailty or prefrailty as determined by a comprehensive geriatric assessment. Our results demonstrated that osteoporosis and sarcopenia, as determined by CTderived body composition analysis, were common in both genders and might be able to discern frail patients from prefrail patients, especially in females where the relationship between frailty and sarcopenia or osteoporosis reached statistical significance. These relationships did not reach statistical significance in males, most likely due to a smaller cohort size. Interestingly, although visceral obesity was not associated with frailty in both groups, BMI and subcutaneous adipose tissue at L3 level were statistically significantly lower in male frail group compared to the prefrail group, and BMI showed strong correlation with subcutaneous area at L3 level in both genders. 
Table 3 Correlation table between CT-derived body composition parameters and laboratory albumin measurement stratified by gender. Asterisk (*) represents statistically significant association with $p<0.05$

\begin{tabular}{|c|c|c|c|c|c|c|}
\hline Female & L3 HU & SMI & Albumin & VAT & SAT & $\mathrm{BMI}$ \\
\hline \multicolumn{7}{|l|}{ L3 HU } \\
\hline SMI & $0.36 *$ & & & & & \\
\hline Albumin & $0.23^{*}$ & $0.28 *$ & & & & \\
\hline VAT & 0.02 & $0.27^{*}$ & $0.18^{*}$ & & & \\
\hline SAT & $0.20 *$ & $0.36^{*}$ & $0.25^{*}$ & $0.67 *$ & & \\
\hline BMI & $0.23 *$ & $0.51 *$ & $0.33 *$ & $0.69 *$ & $0.82 *$ & \\
\hline Male & L3 HU & SMI & Albumin & $\begin{array}{c}\text { Visceral } \\
\text { area }\end{array}$ & $\begin{array}{c}\text { Subcutaneou } \\
\text { s area }\end{array}$ & BMI \\
\hline \multicolumn{7}{|l|}{ L3 HU } \\
\hline SMI & 0.21 & & & & & \\
\hline Albumin & $0.32 *$ & 0.26 & & & & \\
\hline VAT & 0.05 & 0.21 & 0.14 & & & \\
\hline SAT & 0.09 & $0.34 *$ & 0.06 & $0.68^{*}$ & & \\
\hline BMI & 0.18 & 0.59* & 0.29* & $0.67 *$ & 0.78* & \\
\hline
\end{tabular}

Assessment of frailty is important for both clinical and health care policy-making purposes. In trauma, frailty assessment may lead to identification of patients at risk for adverse outcomes should a surgical procedure be performed. For instance, frailty has been shown to be significantly associated with mortality in a large cohort of emergency general surgery admission including both high- and low-risk procedures, raising the importance of preoperative frailty assessment [34]. Assessment of frailty has gained a particular importance during COVID-19 pandemic where the possibility of rationing resources such as ventilator machines has been discussed. Frailty was highlighted as an important tool to address this possibility due to its predictive power of disease outcome which was superior compared to age or comorbidities alone [35]. In terms of health care policy, knowledge of the prevalence of frailty in a given community may play a role in resource distribution, such as funding of the geriatrics department and tailoring range of services available.

While elderly prevalence is projected to continue to increase, estimated to constitute $21 \%$ of the US population by 2030 , the number of geriatric providers per older adult is expected to be almost halved by 2030 compared to the 1980s [36]. Given that elderly patients with a complex medical profile or trauma history frequently undergo CT examinations, with ongoing annual growth in $\mathrm{CT}$ imaging utilization, it is likely that $\mathrm{CT}$ imaging will be much more available than a geriatric appointment [37]. Utilization of information gathered from CT imaging already available in a patient's medical chart represents an attractive strategy for flagging patients at risk for frailty who may benefit 
from a comprehensive geriatric evaluation, and thus be of a significant benefit to society at no additional cost.

On a larger scale, this study serves as a gateway to personalized medicine. It may be viewed as a window to peer to the possibilities of using CT-derived markers of body composition to assess patient's well-being and supplement radiology reports with additional information that may alter patient's treatment or qualify patient for a geriatric workup and assistance with activities of daily living. This analysis will be attributed to the radiologist interpreting the study and will be integrated with the final report, which will provide valuable information regarding patient's health at no extra cost. Further, such information highlights the important role of radiology in the field of medicine and patient care and solidifies its relationship with the field of orthopedics and geriatrics.

It is important to highlight the notion that in this study, comparison was made between frail and prefrail population and it is likely that the association between frailty and sarcopenia will only be magnified for both genders when compared to a healthy geriatric population. For example, the median SMI for both frail women and men corresponded to approximately 25 th percentile or lower of the age-matched SMI reference curve obtained in a recent population study employing automated CT-derived body composition analysis where patients with a diagnosis or major cardiovascular disease or malignancy were excluded from the study [21]. Although the strength between imaging-derived sarcopenia and frailty was reduced when adjusted for age, using this approximation to a relatively healthy adult population, this study suggests that CTderived diagnosis of sarcopenia is common in both frail men and women and will likely serve as a useful screening tool in identifying frail patients and separating them from their healthy peers.

Similarly, given that this study shows that there may be a difference in bone attenuation even between prefrail and frail patients, this difference will likely be only augmented when compared to healthy elderly peers. Additionally, it is worth pointing out that most of the previous studies on frailty and osteoporosis were focusing on women, with little known about men. This study demonstrated that osteoporosis in frail men reached $43.1 \%$ and thus represents an attractive point of intervention as osteoporosis is frequently underdiagnosed and undertreated, especially in men [38].

Hypoalbuminemia was common in frail groups of both genders, although its association with frailty did not reach statistical significance. Hypoalbuminemia has been associated with a decline in muscle strength in the elderly, while SMI addresses a decline in muscle mass [39]. Further research is necessary to elucidate the usefulness of adding laboratory findings such as hypoalbuminemia in the automated frailty diagnosis given that it may provide complimentary information regarding the state of sarcopenia in a frail patient.

Although visceral obesity did not demonstrate an association with frailty, females and males showed different trends in the adipose tissue distribution, and SAT and BMI were statistically significantly lower in the male frail group compared to the prefrail group. Given these findings, it is likely that future frailty detection algorithm development will be gender specific. It is also likely that changes in BMI and adipose tissue distribution, particularly in subcutaneous adipose tissue distribution in men, may be more helpful in frailty detection rather than a specific snapshot of adipose tissue distribution in a given patient at a single point in time.

There were several limitations to this study. Ideally, the control group would be composed of age-matched patients who would be classified as very fit, exhibiting no signs of frailty and have corresponding abdominal CT performed within the same time frame. However, such patients do not get routinely evaluated by geriatric services or undergo CT imaging. Future research may include the prospective recruitment of such patients. Frailty also represents a dynamic clinical condition where pathogenesis includes psychological and social functioning in addition to physical functioning [40, 41]. CT-based assessment of frailty can only approximate physical functioning. One should also consider probable limitations imposed by man-made body composition cutoff values, particularly of sarcopenia, which were not vigorously verified across the older populations without cancer. It would also be advantageous to utilize numerical FI score instead of CFS for correlation of frailty with CT-derived markers of body composition once there are enough patients with prospective FI score assignment. Of note, while our current algorithm does not automatically extract bone attenuation, an upgraded version of the model with both automated SMI and bone attenuation information will be more useful.

In summary, this proof-of-concept study suggests promising associations between CT-derived body composition parameters and frailty and serves as an important foundation in the development of automated geriatric frailty screening algorithm based on abdominal CT-imaging. While a formal frailty assessment cannot be replaced, the use of automated detection algorithms may be able to alert clinicians to patients at higher risk of being frail. Future research is necessary to continue exploring associations between body composition and frailty, and it is likely that a combination of multiple body composition imaging parameters as well as laboratory values and changes in BMI and adipose tissue distribution over time will be necessary for the creation of a sensitive and specific CT imaging-based frailty detection algorithm. 


\section{References}

1. Rockwood K, Mitnitski A. Frailty defined by deficit accumulation and geriatric medicine defined by frailty. Clin Geriatr Med [Internet]. 2011;27(1):17-26. Available from: https://doi.org/ 10.1016/j.cger.2010.08.008

2. Fried LP, Tangen CM, Walston J, Newman AB, Hirsch C, Gottdiener J, et al. Frailty in older adults: evidence for a phenotype. Journals Gerontol Ser A Biol Sci Med Sci. 2001;56(3):M146-56.

3. Schuurmans H, Steverink N, Lindenberg S, Frieswijk N, Slaets JPJ. Old or frail: what tells us more? Journals Gerontol Ser A Biol Sci Med Sci. 2004;59(9):M962-5.

4. Krishnan M, Beck S, Havelock W, Eeles E, Hubbard RE, Johansen A. Predicting outcome after hip fracture: using a frailty index to integrate comprehensive geriatric assessment results. Age Ageing. 2014;43(1):122-6.

5. Dent E, Morley JE, Cruz-Jentoft AJ, Woodhouse L, RodríguezMañas L, Fried LP, et al. Physical frailty: ICFSR international clinical practice guidelines for identification and management. J Nutr Heal Aging. 2019;23(9):771-87.

6. Robinson TN, Wu DS, Stiegmann GV, Moss M. Frailty predicts increased hospital and six-month healthcare cost following colorectal surgery in older adults. Am J Surg. 2011;202(5):511-4.

7. Makary MA, Segev DL, Pronovost PJ, Syin D, Bandeen-Roche $\mathrm{K}$, Patel P, et al. Frailty as a predictor of surgical outcomes in older patients. J Am Coll Surg. 2010;210(6):901-8.

8. Hall DE, Arya S, Schmid KK, Carlson MA, Lavedan P, Bailey TL, et al. Association of a frailty screening initiative with postoperative survival at 30,180, and 365 days. JAMA Surg. 2017;152(3):233-40.

9. Oakland K, Nadler R, Cresswell L, Jackson D, Coughlin PA. Systematic review and meta-analysis of the association between frailty and outcome in surgical patients. Ann R Coll Surg Engl. 2016;98(2):80-5.

10. Joseph B, Pandit V, Zangbar B, Kulvatunyou N, Hashmi A, Green DJ, et al. Superiority of frailty over age in predicting outcomes among geriatric trauma patients: a prospective analysis. JAMA Surg. 2014;149(8):766-72.

11. Lin HS, Watts JN, Peel NM, Hubbard RE. Frailty and post-operative outcomes in older surgical patients: a systematic review. BMC Geriatr. 2016;16(1):157.

12. O'Caoimh R, Galluzzo L, Rodríguez-Laso Á, Van Der Heyden J, Ranhoff AH, Lamprini-Koula M, et al. Prevalence of frailty at population level in European ADVANTAGE Joint Action Member States: a systematic review and meta-analysis. Ann Ist Super Sanita. 2018;54(3):226-38.

13. Clegg A, Young J, Iliffe S, Rikkert MO, Rockwood K. Frailty in elderly people. Lancet (London, England). 2013;381(9868):752-62.

14. Stewart RM, Rotondo MF, Nathens AB, Neal M, Caden-Price C, Lynch J, et al. Trauma Quality Programs NTDB/TQIP Staff [Internet]. [cited 2021 Jan 19]. Available from: www.ntdb.org.

15. Cooper L, Loewenthal J, Frain LN, Tulebaev S, Cardin K, Hshieh TT, et al. From research to bedside: incorporation of a CGA-based frailty index among multiple comanagement services. J Am Geriatr Soc. 2021 Sep 13. Online ahead of print. Available from: https://doi.org/10.1111/jgs.17446

16. Rockwood $\mathrm{K}$, Theou $\mathrm{O}$. Using the clinical frailty scale in allocating scarce health care resources. Can Geriatr J [Internet]. 2020 Sep 1;23(3):210-5. Available from: https://pubmed.ncbi. nlm.nih.gov/32904824

17. Shrier W, Dewar C, Parrella P, Hunt D, Hodgson LE. Agreement and predictive value of the Rockwood Clinical Frailty Scale at emergency department triage. Emerg Med J [Internet]. 2020
Nov 10;emermed-2019-208633. Available from: http://emj.bmj. com/content/early/2020/11/09/emermed-2019-208633.abstract

18. Idoate F, Cadore EL, Casas-Herrero A, Zambom-Ferraresi F, Marcellán T, de Gordoa AR, et al. Adipose tissue compartments, muscle mass, muscle fat infiltration, and coronary calcium in institutionalized frail nonagenarians. Eur Radiol. 2015;25(7):2163-75.

19. Kaplan SJ, Pham TN, Arbabi S, Gross JA, Damodarasamy M, Bentov I, et al. Association of radiologic indicators of frailty with 1-year mortality in older trauma patients: opportunistic screening for sarcopenia and osteopenia. JAMA Surg. 2017;152(2):1-8.

20. Bredella MA. Sex differences in body composition. In: Advances in Experimental Medicine and Biology. 2017; 1043:9-27

21. Magudia K, Bridge CP, Bay CP, Babic A, Fintelmann FJ, Troschel FM, et al. Population-scale CT-based body composition analysis of a large outpatient population using deep learning to derive age-, sex-, and race-specific reference curves. Radiology. 2021;298(2):319-29.

22. Bridge CP, Rosenthal M, Wright B, Kotecha G, Fintelmann F, Troschel F, et al. Fully-automated analysis of body composition from CT in cancer patients using convolutional neural networks. In: Stoyanov D, Taylor Z, Sarikaya D, McLeod J, González Ballester MA, Codella NCF, et al., editors. OR 20 Context-Aware Operating Theaters, Computer Assisted Robotic Endoscopy, Clinical Image-Based Procedures, and Skin Image Analysis. Cham: Springer International Publishing; 2018. p. 204-13.

23. Blodgett J, Theou O, Kirkland S, Andreou P, Rockwood K. Frailty in NHANES: Comparing the frailty index and phenotype. Arch Gerontol Geriatr [Internet]. 2015;60(3):464-70. Available from: https://www.sciencedirect.com/science/article/pii/S016749431 5000242

24. Akirov A, Masri-Iraqi H, Atamna A, Shimon I. Low albumin levels are associated with mortality risk in hospitalized patients. Am J Med [Internet]. 2017;130(12):1465.e11-1465.e19. Available from: https://doi.org/10.1016/j.amjmed.2017.07.020

25. Martin L, Birdsell L, MacDonald N, Reiman T, Clandinin MT, McCargar LJ, et al. Cancer cachexia in the age of obesity: Skeletal muscle depletion is a powerful prognostic factor, independent of body mass index. J Clin Oncol. 2013;31(12):1539-47.

26. Lee SJ, Graffy PM, Zea RD, Ziemlewicz TJ, Pickhardt PJ. Future osteoporotic fracture risk related to lumbar vertebral trabecular attenuation measured at routine body CT. J Bone Miner Res. 2018;33(5):860-7.

27. Williams MJ, Hunter GR, Kekes-Szabo T, Trueth MS, Snyder $\mathrm{S}$, Berland L, et al. Intra-abdominal adipose tissue cut-points related to elevated cardiovascular risk in women. Int $\mathrm{J}$ Obes. 1996;20(7):613-7.

28. Hunter GR, Snyder SW, Kekes-Szabo T, Nicholson C, Berland L. Intra-abdominal adipose tissue values associated with risk of possessing elevated blood lipids and blood pressure. Obes Res. 1994;2(6):563-8.

29. Murray T, Williams D, Lee MJ. Osteoporosis, obesity, and sarcopenia on abdominal CT: a review of epidemiology, diagnostic criteria, and management strategies for the reporting radiologist. Abdom Radiol. 2017;42(9):2376-86. https://doi.org/10.1007/ s00261-017-1124-5.

30. Cruz-Jentoft AJ, Bahat G, Bauer J, Boirie Y, Bruyère O, Cederholm T, et al. Sarcopenia: revised European consensus on definition and diagnosis. Age Ageing. 2019;48(1):16-31.

31. Morley JE, Anker SD, von Haehling S. Prevalence, incidence, and clinical impact of sarcopenia: facts, numbers, and epidemiologyupdate 2014. J Cachexia Sarcopenia Muscle. 2014;5(4):253-9.

32. Morley JE, von Haehling S, Anker SD, Vellas B. From sarcopenia to frailty: a road less traveled. J Cachexia Sarcopenia Muscle. 2014;5(1):5-8.

33. Sambrook PN, Cameron ID, Chen JS, Cumming RG, Lord $\mathrm{SR}$, March LM, et al. Influence of fall related factors and bone 
strength on fracture risk in the frail elderly. Osteoporos Int. 2007;18(5):603-10.

34. Castillo-Angeles M, Cooper Z, Jarman MP, Sturgeon D, Salim A, Havens JM, Association of frailty with morbidity and mortality in emergency general surgery by procedural risk level. JAMA Surg,. 1;156(1):68-74. Available from: 2021. https://doi.org/10.1001/ jamasurg.2020.5397.

35. Hewitt J, Carter B, Vilches-Moraga A, Quinn TJ, Braude P, Verduri A, et al. The effect of frailty on survival in patients with COVID-19 (COPE): a multicentre, European, observational cohort study. Lancet Public Heal [Internet]. 2020 Aug 1;5(8):e444-51. Available from: https://doi.org/10.1016/S24682667(20)30146-8

36. Morley JE. The future of geriatrics. Journal of Nutrition, Health and Aging. 2020;24:1-2.

37. Smith-Bindman R, Kwan ML, Marlow EC, Theis MK, Bolch W, Cheng SY, et al. Trends in use of medical imaging in US Health Care Systems and in Ontario, Canada, 2000-2016. JAMA. 2019;322(9):843-56.
38. Qaseem A, Forciea MA, McLean RM, Denberg TD. Treatment of low bone density or osteoporosis to prevent fractures in men and women: a clinical practice guideline update from the American college of physicians. Ann Intern Med. 2017;166(11):818-39.

39. Schalk BWM, Deeg DJH, Penninx BWJH, Bouter LM, Visser M. Serum albumin and muscle strength: a longitudinal study in older men and women. J Am Geriatr Soc. 2005;53(8):1331-8.

40. Xue QL. The Frailty Syndrome: Definition and Natural History. Clin Geriatr Med. 2011;27(1):1-15.

41. Collard RM, Boter H, Schoevers RA, Oude Voshaar RC. Prevalence of frailty in community-dwelling older persons: a systematic review. J Am Geriatr Soc. 2012;60(8):1487-92.

Publisher's note Springer Nature remains neutral with regard to jurisdictional claims in published maps and institutional affiliations. 